\title{
The Usefulness of the Endonasal Incisional Approach for the Treatment of Nasal Bone Fracture
}

\author{
Hyo Seong Kim, Hyeun Woo Suh, Ki Young Ha, Boo Yeong Kim, Tae Yeon Kim \\ Department of Plastic and Reconstructive Surgery, Bundang Jesaeng General Hospital, Seongnam, Korea
}

Background Among all facial fractures, nasal bone fractures are the most common, and they have been reduced by closed reduction (CR) for a long time. But several authors have reported suboptimal results when using $\mathrm{CR}$, and the best method of nasal bone reduction is still being debated. We have found that indirect open reduction (IOR) through an endonasal incisional approach is a useful method for more accurate reduction of the nasal bone.

Methods A retrospective chart review was performed of 356 patients who underwent reduction of a nasal bone fracture in our department from January, 2006, to July, 2011. We treated 263 patients with IOR. We assessed patients' and doctors' satisfaction with surgical outcomes after IOR or CR. We evaluated the frequency of nasal bleeding owing to mucosal injury, and followed the surgical outcomes of patients who had simultaneous dorsal augmentation rhinoplasty.

Results According to the analysis of the satisfaction scores, both patients and doctors were significantly more satisfied in the IOR group than the CR group $(P<0.05)$. Mucosal injury with nasal bleeding occurred much less in the IOR group (5.3\%) than the CR group (12.9\%). Dorsal augmentation rhinoplasty with IOR was performed simultaneously in 34 cases. Most of them (31/34) showed satisfaction with the outcomes.

Conclusions IOR enables surgeons to manipulate the bony fragment directly through the endonasal incisional approach. However, we propose that CR is the proper technique for patients under 16 and for those with comminuted nasal bone fractures because submucosal dissection in IOR can damage the growth or circulation of nasal bone.

Keywords Nasal bone / Fractures / Injuries
Correspondence: Tae Yeon Kim Department of Plastic and Reconstructive Surgery, Bundang Jesaeng General Hospital, 20 Seohyeon-ro 180beon-gil, Bundang-gu, Seongnam 463-774, Korea

Tel: +82-31-779-0281

Fax: +82-31-779-0164

E-mail: khs1002@dmc.or.kr
This article was presented at the 69th Congress of the Korean Society of Plastic and Reconstructive Surgeons on Nov 11-13, 2011, in Seoul, Korea.

No potential conflict of interest relevant to this article was reported.

\section{INTRODUCTION}

Because the nose is prominently located in the center of the face, nasal bone fractures are the most frequent facial bone fracture and the third most frequent fracture in the body [1]. Despite the fact that there are various characteristics and types of such fractures, closed reduction with simple manipulation has been considered the conventional choice of treatment for all of them
[2]. However, in spite of adequate closed reduction (CR) treatment, the incidence of posttraumatic nasal deformity remains high, and several authors report suboptimal results. Therefore, the best method of reduction of nasal bone fractures is still being debated $[1,2]$. Since the majority of cases of nasal bone reduction in the past have been attempted under local anesthesia, the simpler closed reduction has conventionally been performed. However, since most of nasal bone reduction operations are now 
being performed under general anesthesia, it is possible to undertake more aggressive and efficient procedures. Burm and $\mathrm{Oh}$ [3] introduced a more direct technique for nasal bone reduction using an endonasal incisional approach, known as indirect open reduction (IOR) for proper reduction of nasal bone.

We have found that the IOR technique through an endonasal incisional approach is a useful method for more accurate and satisfactory reduction of the nasal bone. To determine the efficacy of the IOR technique in nasal bone reduction, we evaluated patient and physician scores on satisfaction with postsurgical results, the risk of nasal mucosa injury, and the usefulness of simultaneous dorsal augmentation rhinoplasty for cosmetic purposes.

\section{METHODS}

\section{Patients}

A retrospective chart review was performed of 356 patients who had undergone reduction of nasal bone fracture in our department during a 5.5-year period, from January, 2006 to July, 2011. The operations were performed by four plastic surgeons on 273 male and 83 female patients from 2 to 80 years with a mean age of 29.0 years. CR was performed by three surgeons (A, C, D) in 93 patients, and IOR using an endonasal incisional approach was performed by three surgeons (A, B, C) in 263 patients (Table 1). Preoperative and postoperative photographs of the patients were taken in the sitting position. All patients were evaluated before and after surgery by plain radiography (Waters view, nasal bone view laterally from both sides). Preoperative facial bone computed tomography $(\mathrm{CT})$ scan was routinely taken to visualize the fracture shape for all patients, but only in a few patients, were we able to perform a facial bone $\mathrm{CT}$ after surgery due to insurance regulations.

\section{IOR surgical technique using an endonasal incisional approach}

Under general anesthesia, CR was performed by the ordinary surgical method using Asch forceps or the Nelaton-tipped long Kelly. The indirect open reduction method using the endonasal incisional approach is detailed below.

After local infiltration into the mucous membrane in the boundary of the nasal cartilage, an intra- or intercartilaginous incision

Table 1. Cases of reduction of nasal bone fracture by 4 plastic surgeons

\begin{tabular}{|lrrrrr|}
\hline Method & A & B & C & D & Total \\
\hline Closed reduction & 42 & 0 & 21 & 30 & $93(26.1 \%)$ \\
Indirect open reduction & 157 & 104 & 2 & 0 & $263(73.9 \%)$ \\
\hline A, B, C, D, four plastic surgeons. & & & & \\
\hline
\end{tabular}

was carried out in the nasal cavity, and the subcutaneous tissue was undermined from the cartilaginous layer. By using the periosteal elevator reaching beyond the caudal margin of the nasal bone, submucosal dissection was performed to expose the dislocated bone pieces caused by the fracture. The fractured nasal bone segments were reduced in the original position with a periosteal elevator by feeling with the hands without visualization (Fig. 1). Thereafter, the Nelaton-tipped long Kelly was used to perform additional gentle reduction as a closed reduction method. To protect and support the fractured nasal bones in their reduced positions, nasal packing with petrolatum gauze and Multicel sponge was kept in position for 3 to 5 days by surgeons A, C, and D (159 cases) including the cases of simultaneous dorsal augmentation rhinoplasty. Surgeon B (104 cases) used Multicel and K-wire fixation to support the fractured bone. The nose was packed with Multicel sponge for 3 days and 2 or $3 \mathrm{~K}$-wires were removed 5 days after the operation. All the surgeons applied external aluminum splints after the operation, and these splints were left in position for 2 to 3 weeks.

\section{Assessment methods}

\section{Comparison of satisfaction after CR and IOR by patients and} doctors

Three or 4 weeks postoperatively, patients' and doctors' satisfaction with the surgical outcome after IOR or CR was assessed by questionnaires in which items were rated with a score ranging from 1 to 5 ( 1 , very dissatisfied; 2 , dissatisfied; 3 , fair; 4 , satisfied; 5 , very satisfied). The mean of the scores for all the items was calculated. The patients' degree of satisfaction was measured by satisfaction with their external nasal shape, such as depression, deviation, or protrusion, and by the degree of reduction of discomfort symptoms such as pain, edema, crepitation, or nasal stuffiness after the surgery (Table 2). The doctors' degree of

Fig. 1. Surgical technique of indirect open reduction (IOR) through endonasal incisional approach

(A) An intercartilaginous or intracartilaginous incision was made. (B) By using a periosteal elevator reaching to the caudal margin of the nasal bone, a submucosal pocket dissection was performed. The fractured nasal bone segments were reduced in the original position with the periosteal elevator by feeling with the hands without visualization.

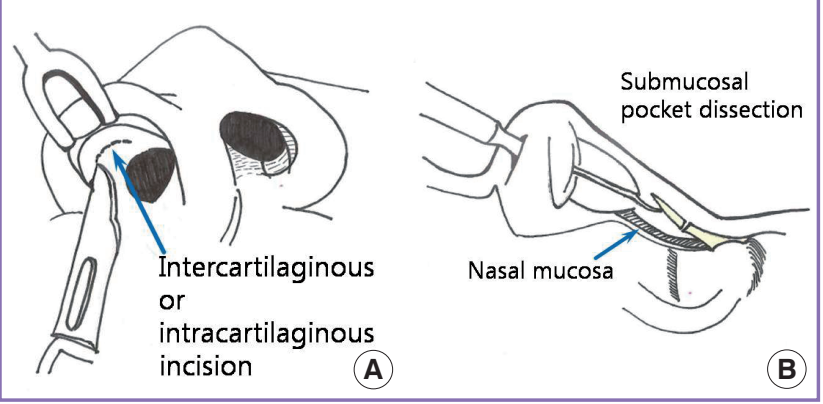


satisfaction was measured by two residents who independently viewed the photographs to evaluate the nasal external symmetry and dorsal contour, and postsurgical plain radiography (lateral nasal view and Waters view) as objective data (Table 3 ).

Frequency of nasal bleeding due to mucosal injury

There are often cases of nasal bleeding during surgery or postoperative epistaxis caused by damage to the mucous membrane in the nasal cavity during the nasal bone reduction procedure. We analyzed the frequency of nasal bleeding with IOR and CR operations.

Delayed reduction of nasal bone fracture after 1 or 2 weeks of trauma

It is known that reduction of the fractured nasal bone should be performed in the first week after trauma for bony healing and union. We attempted nasal bone reduction after 1 or 2 weeks of trauma using IOR.

\section{Simultaneous dorsal augmentation rhinoplasty}

In 34 patients ( 25 male, 9 female) who received reduction due to nasal bone fractures, dorsal augmentation rhinoplasty was performed simultaneously for cosmetic purposes. After bony reduction through the endonasal approach, rhinoplasty was performed through the same incision with a nasal septal cartilage graft or Alloderm to augment a low nasal dorsum. We did not use a silastic nasal implant or perform delicate tip plasty, because the nose had acute trauma injury and there is a high risk of inflammation after trauma injury. We followed the surgical outcome of patients who had undergone simultaneous dorsal augmentation rhinoplasty.

\section{RESULTS}

\section{Cases who had IOR operations}

Two hundred sixty-three patients, among whom were $171 \mathrm{pa}-$ tients with lateral wall fracture, 72 patients with nasal bone tip

Table 2. The standard questionnaire for estimation of satisfaction by patients

\begin{tabular}{|lccccc|}
\hline Satisfaction scale & Very dissatisfied & Dissatisfied & Fair & Satisfied & Very satisfied \\
\hline External nasal shape & 1 & 2 & 3 & 4 & 5 \\
Discomfort symptoms & 1 & 2 & 3 & & \\
\hline External nasal shape: depression, deviation or protrusion. & & & & \\
Discomfort symptoms: pain, edema, crepitation or nasal stuffiness. & & & \\
\hline
\end{tabular}

\section{Table 3. The standard questionnaire for estimation of satisfaction by doctors}

\begin{tabular}{|lccccc|}
\hline Satisfaction scale & Very dissatisfied & Dissatisfied & Fair & Satisfied & Very satisfied \\
\hline Photograph & 1 & 2 & 3 & 4 & 5 \\
Plain radiography & 1 & 2 & 3 & & \\
\hline $\begin{array}{l}\text { Photograph: nasal symmetry, dorsal contour. } \\
\text { Plain radiography: lateral nasal view, Waters view. }\end{array}$ & & & & \\
\hline
\end{tabular}

\section{Fig. 2. Case of depressed lateral nasal bone fracture}

(A) CT scan view of nasal bone fracture. Preoperative view of the depressed lateral nasal bone fracture. (B) CT scan view of nasal bone fracture. Postoperative view of depressed lateral nasal bone fracture. (C) Schematic illustration of cross-sectional nasal view showing the superiority of indirect open reduction (IOR) for the reduction of depressed lateral nasal bone fracture compared to closed reduction (CR).
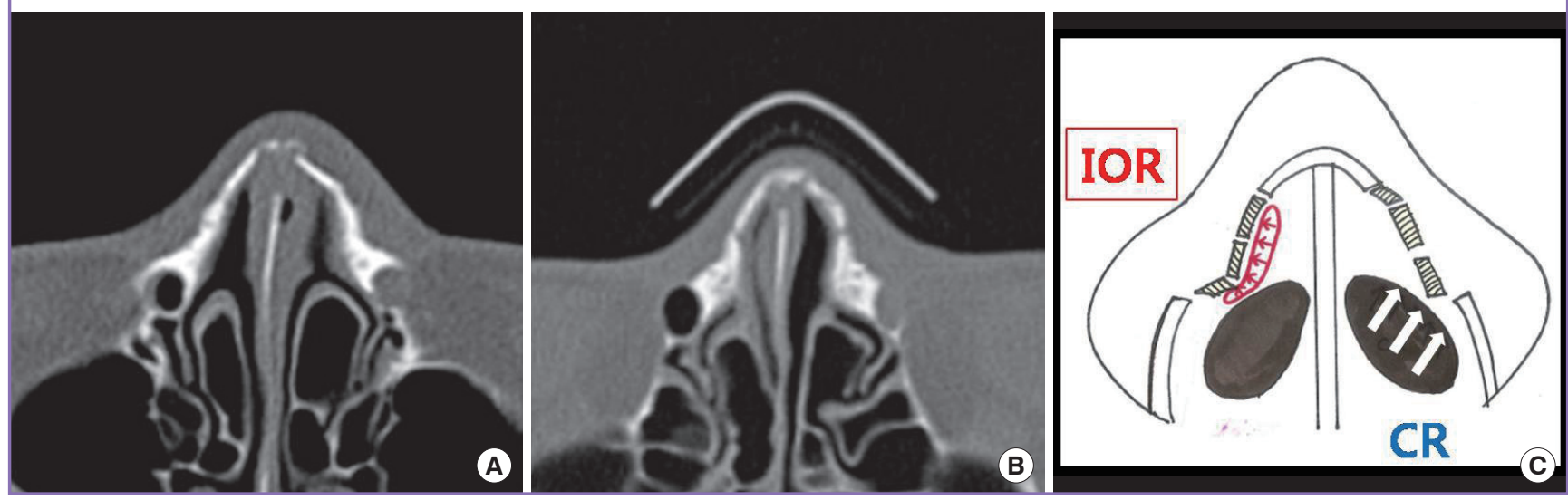


\section{Fig. 3. Case of depressed nasal tip fracture}

(A) X-ray view of nasal bone fracture. Preoperative view of depressed nasal tip fracture. (B) X-ray view of nasal bone fracture. Postoperative view of depressed nasal tip fracture. (C) Schematic illustration of cross-sectional nasal view showing the superiority of indirect open reduction (IOR) for the reduction of depressed nasal tip fracture compared to closed reduction (CR).
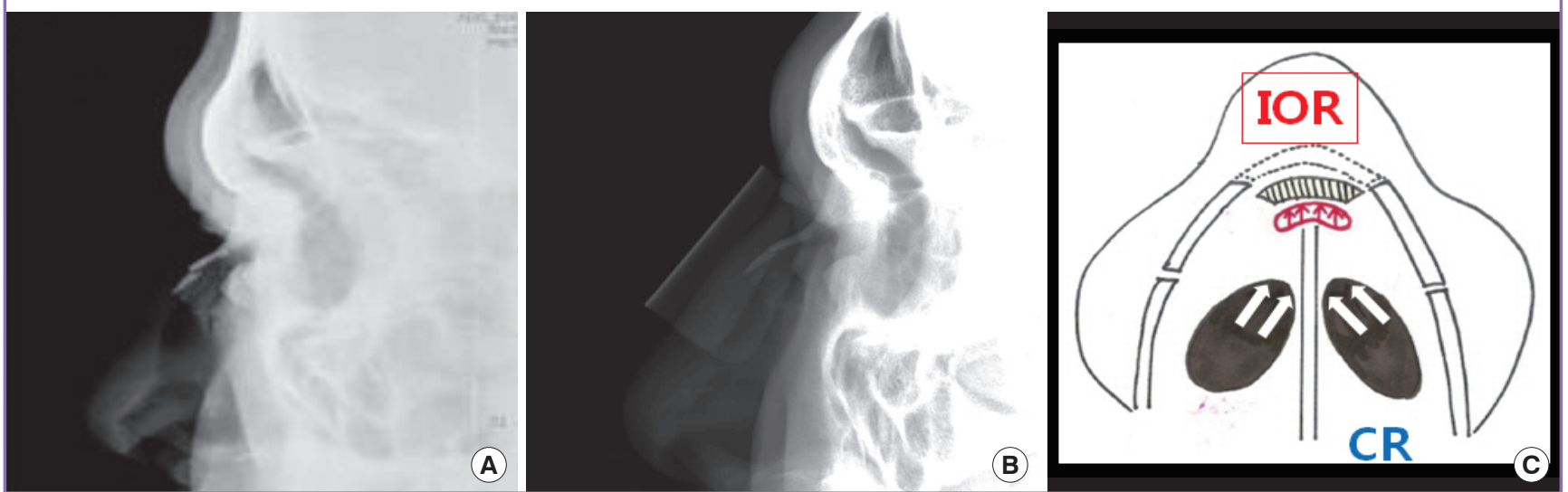

\section{Fig. 4. Case of focal buckling fracture of the right lateral wall}

(A) CT scan view of nasal bone fracture. Preoperative view of focal buckling fracture of the right lateral wall. (B) CT scan view of nasal bone fracture. Postoperative view of focal buckling fracture of the right lateral wall. (C) Schematic illustration of cross-sectional nasal view showing the superiority of indirect open reduction (IOR) for the reduction of focal buckling fracture of nasal bone compared to closed reduction (CR).
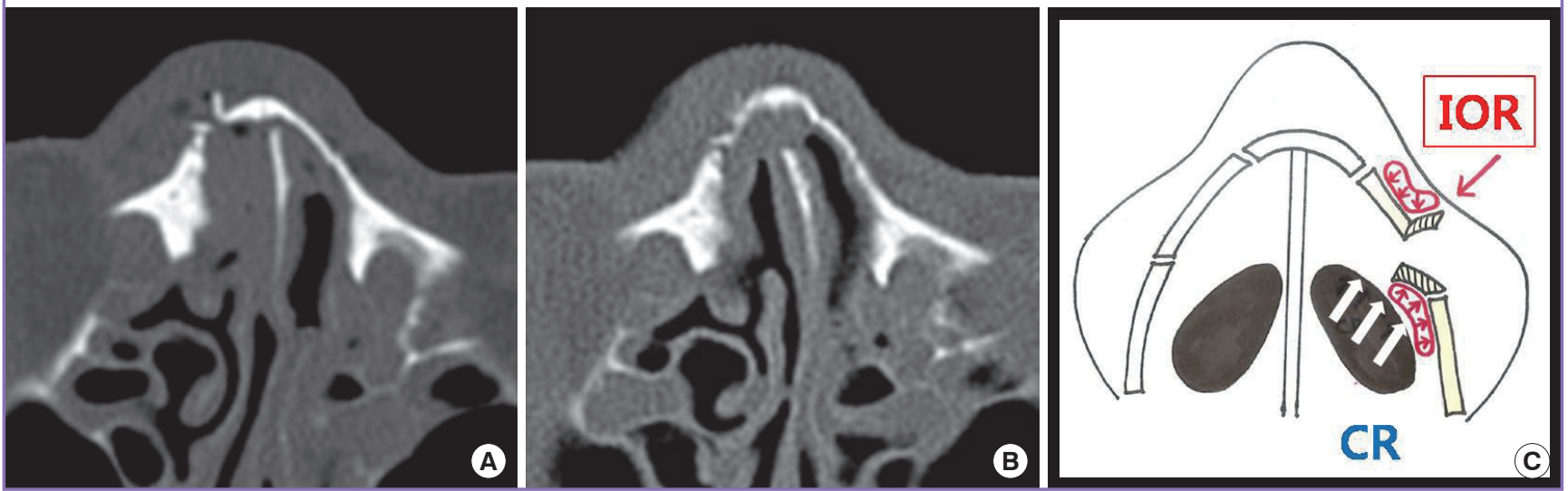

Table 4. Comparison of satisfaction according to the methods of reduction in nasal bone fracture by patients and doctors

\begin{tabular}{|lccr|}
\hline Satisfaction scale & CR $(\mathbf{n}=\mathbf{9 3})$ & IOR $(\mathbf{n}=\mathbf{2 6 3})$ & t-test \\
\hline Patients & $3.8 \pm 0.61$ & $4.2 \pm 0.69$ & $\mathrm{P}<0.05$ \\
Doctors & $3.6 \pm 0.50$ & $4.3 \pm 0.71$ & $\mathrm{P}<0.05$ \\
\hline $\begin{array}{l}\text { CR, closed reduction; IOR, indirect open reduction. } \\
\text { Values are mean } \pm S D .\end{array}$ \\
\hline
\end{tabular}

fracture, and 20 patients with focal individual fracture, underwent indirect open reduction through an endonasal incisional approach by 3 plastic surgeons (Figs. 2-4).

\section{Assessment results}

Comparison of satisfaction after CR and IOR by patients and doctors

After reduction, the satisfaction scores of patients were 4.2 in

Table 5. Cases of nasal mucosa injury with epistaxis in reduction
\begin{tabular}{|lcc|}
\hline Group & CR $(\mathrm{n}=\mathbf{9 3})$ & IOR $(\mathrm{n}=\mathbf{2 6 3})$ \\
\hline Nasal mucosa injury or epistaxis & $12(12.9 \%)$ & $14(5.3 \%)$ \\
\hline CR, closed reduction; IOR, indirect open reduction. & \\
\hline
\end{tabular}

the IOR group and 3.8 in the CR group. The satisfaction score of doctors was found to be 4.3 in the IOR group and 3.6 in the CR group. Both patients and doctors were significantly more satisfied with the IOR technique than with $\mathrm{CR}(\mathrm{P}<0.05)$ (Table 4).

\section{Frequency of nasal bleeding due to mucosal injury}

Mucosal injury with nasal bleeding occurred much less in the IOR group (5.3\%) than the CR group (12.9\%) (Table 5). 
Fig. 5. Case of simultaneous dorsal augmentation rhinoplasty

(A) A 25-year-old male presented with nasal bone fracture with a low nasal dorsum. He underwent indirect open reduction (IOR) of the nasal bone fracture and simultaneous dorsal augmentation rhinoplasty with an Alloderm graft. There were no postoperative complications and the patient showed satisfaction at 1-year follow-up. Preoperative photo. (B). One-year postoperative photo. (C). Preoperative X-ray view. (D) Postoperative X-ray view.
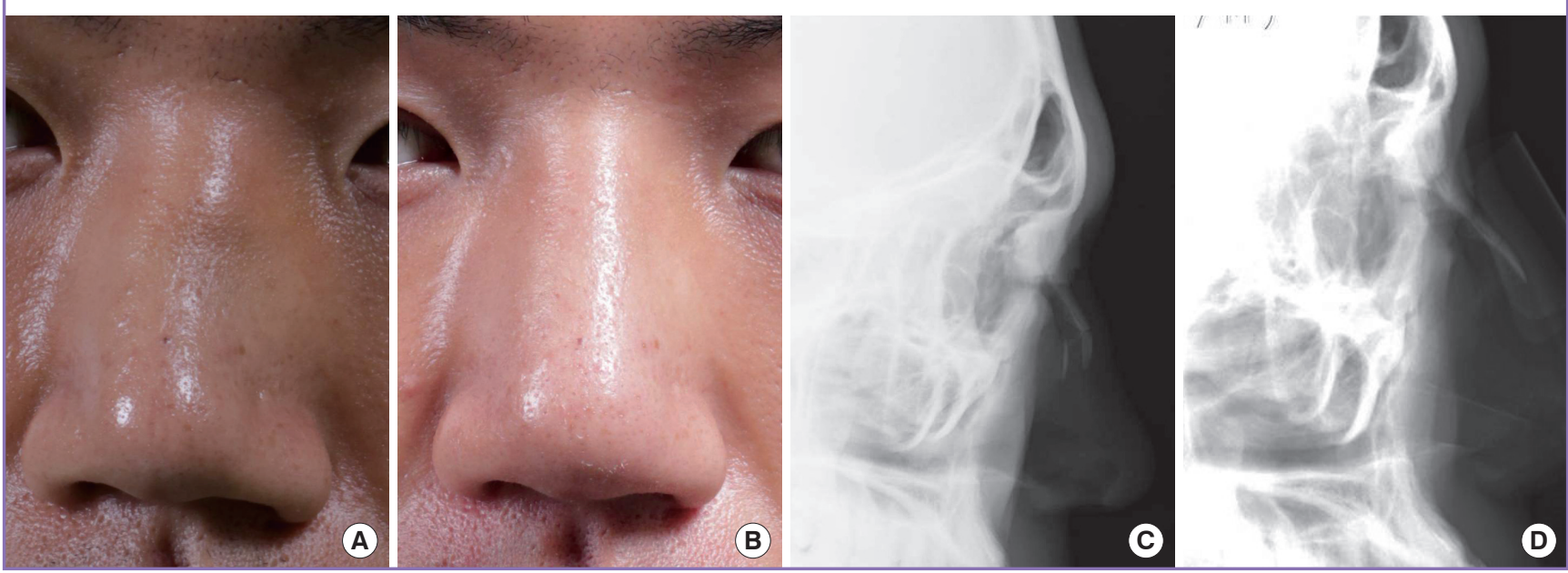

Table 6. Satisfaction of patients who had simultaneous dorsal augmentation rhinoplasty

\begin{tabular}{|lcccccc|}
\hline Group & Very satisfied & Satisfied & Fair & Dissatisfied & Very dissatisfied & Total \\
\hline Number of patients (\%) & $12(35.4)$ & $19(55.8)$ & $2(5.9)$ & $1(2.9)$ & $0(0)$ & 34 \\
\hline
\end{tabular}

\section{Delayed reduction of nasal bone fracture after 1 or 2 weeks of trauma}

We attempted to reduce the fractured nasal bone after 1 or 2 weeks of trauma in 19 patients using IOR. Although there was significant resistance against the reduction, it was possible to restore the fractured bony fragment into its original place in all patients. Both patients and doctors were contented with the postoperative results.

\section{Simultaneous dorsal augmentation rhinoplasty}

In 34 cases, we used Alloderm or Surederm in 22 patients, autologous septal cartilage in 11 patients, and both of them in 1 patient (Fig. 5). 91.2\% of them were satisfied with the postoperative results (very satisfied, 12; satisfied, 19; fair, 2; dissatisfied, 1; very dissatisfied, 0 ) (Table 6).

\section{DISCUSSION}

In general, closed reduction has been widely used as the treatment method for nasal fractures. This is a relatively simple and easy method to perform under local or general anesthesia. However, many authors have reported that a great number of outcomes have not satisfied expectations. Rohrich and Adams [2] reported that posttraumatic nasal deformity requiring subsequent rhinoplasty or septorhinoplasty remained in as many as $50 \%$ of cases $[1,2,4]$.

Tremolet de Villers [5] explained the cause of poor aesthetic outcomes after closed reduction of nasal bone fractures in light of anatomic location and characteristics. Specifically, because the nose is at the center of the face, its deformity is most noticeable after injury and the very thin skin and soft tissue over the nasal protrusion of the face provide only fragile coverage. The strong muscles that can be used to restore a fractured segment into a reduction site are lacking around the nasal bone. Also, in the healing process of nasal bone, fibrosis and scarring have been reported to be severe compared with those in long bones.

The thin nasal bone with a three-dimensional structure, as a property of the nasal structure, tends to break down into numerous pieces when the nasal bone is fractured, and it becomes difficult to restore each fragment to fit its original three-dimensional structure by using simple closed reduction. In 1966, Fry [6] reported the interlocked stress to the cartilage after traumatic injury makes nasal deformity more severe. Because of this, redepression of the fractured segment and nasal deformity is not uncommon after nasal bone reduction.

In particular, there is a limit to $\mathrm{CR}$ in cases of a focally upward displaced fragment fracture or a midline tip fracture. Moreover, attempts to use CR too extremely may cause nasal bleeding due to damage to the nasal mucous membrane or mucosal synechia that may occur after the wound has healed [7]. 
In 1998, Burm and Oh [3] introduced a more aggressive technique than $\mathrm{CR}$ for nasal bone reduction using an endonasal incisional approach, named IOR. Similarly, in most of our cases of IOR through the endonasal approach, the surgeons used the periosteal elevator to undermine the space between the nasal mucosa and fractured bony area, lifting up or reducing the dislocated bone pieces to restore them to their original location. The IOR method, in particular, enables surgeons to feel the midline fractured area of the nasal bone tip, focally displaced fragments which are upwardly or downwardly of the nasal bone with the hand for accurate positioning instead of blunt trial during CR. According to statistical analysis of the satisfaction scores from the present study, both patients and doctors were more satisfied with the surgical outcomes after IOR than CR. This result is due to the fact that IOR allows more accurate reduction since it enables operators to manipulate the fractured bony fragment directly through the submucosal dissection by sufficiently feeling the fractured bone pieces with the hands although they cannot be visually observed.

After IOR of the fractured bone segments, surgeons gently performed CR for final retouching. Since there is no need for excessive strength in these reduction processes, there was almost no danger of nasal bleeding caused by damage to the nasal mucous membrane when compared with CR only.

Usually the proper time for reduction of a nasal bone fracture is within 7 days after trauma. Because the bony union becomes hard by callus formation after this period, it is difficult to reduce the displaced fracture segments after that time. On the other hand, IOR places direct strength on the fractured bone itself; therefore, it is possible to restore the fracture to its original place even 1 or 2 weeks afterward [8].

To keep the reduced nasal bone in its proper position, the authors performed intranasal petrolatumgauze and Multicel sponge packing or K-wire fixation to maintain the restored fragments. For the comparison of the effects of the two fixation methods, more detailed research is required.

Another merit of the IOR operation is that fracture reduction and aesthetic rhinoplasty can be simultaneously performed through the same incision inside the nostril for patients desiring to augment their low nasal dorsum for cosmetic purposes. In dorsal augmentation rhinoplasty, due to irregularity and unstableness of the fractured side, we used Alloderm, Surederm or a cartilage graft of the nasal septum, which is more flexible, instead of silastic implants, which are solid. Because nasal bone fracture is an acute trauma injury, it is difficult to perform detailed and delicate rhinoplasty at the same time. We did not attempt other rhinoplasty, such as tip plasty, due to the high risk of infection or inflammation. As a procedure before the operation, it is required to execute sufficient consultation with patients and to obtain accurate information about the fracture to satisfy the aesthetic desires of the patient in planning the operation [4]. Simultaneous augmentation rhinoplasty was performed in patients with mild nasal bone fracture and moderate or severe dorsal depression. The patients who had comminuted nasal bone fracture, septal fracture, or severe deviated septum were excluded from the indications of simultaneous dorsal augmentation rhinoplasty in spite of the desire of patients. The authors try not to dissect too widely to insert the graft material for augmentation to avoid the deviation of the graft after placement. Also, the insertion level was not on the nasal bone, but in the subcutaneous pocket for better circulation. Among our patients who underwent the IOR method with dorsal augmentation rhinoplasty, the longest postoperative follow-up is 1 year so far. However, we will continue to follow up to observe outcomes over the long term.

Despite our positive results, it is important to note that submucosal dissection in the IOR method may cause damage to the periosteum of the nasal bone. Thus, in patients under 16 years of age, the IOR procedure may harm bone growth. Furthermore, in patients with comminuted nasal bone fracture, the submucosal dissection in the IOR procedure can scatter the comminuted fracture segments that are maintained by the soft tissue into multiple separate pieces and disrupt the blood circulation of the fractured fragments. Therefore, in patients under 16 years of age or with comminuted fracture, CR is considered to be safer and more appropriate than IOR.

In conclusion, IOR can be utilized more efficiently than CR for the treatment of nasal bone fracture in light of the following findings: 1) According to statistical analysis of a satisfaction scale administered by questionnaire, the patients and surgeons of the IOR group were more satisfied with their surgical outcomes than those of the CR group $(\mathrm{P}<0.05) .2)$ Mucosal injury with nasal bleeding occurred significantly less in the IOR group (5.3\%) than the CR group (12.9\%). 3) Nasal bone reduction was possible to perform 1 or 2 weeks after fracture when using the IOR method. 4) The IOR method also gave us the advantage of being able to perform simultaneous dorsal augmentation rhinoplasty for cosmetic purposes with good results (31/34).

\section{REFERENCES}

1. Murray JA, Maran AG, Mackenzie IJ, et al. Open v closed reduction of the fractured nose. Arch Otolaryngol 1984;110: 797-802.

2. Rohrich RJ, Adams WP Jr. Nasal fracture management: minimizing secondary nasal deformities. Plast Reconstr Surg 2000;106:266-73. 
3. Burm JS, Oh SJ. Indirect open reduction through intercartilaginous incision and intranasal Kirschner wire splinting of comminuted nasal fractures. Plast Reconstr Surg 1998; 102:342-9.

4. Kim NY, Lee SH, Choi HG, et al. Simultaneous rhinoplasty with fracture reduction in nasal bone fracture. J Korean Soc Plast Reconstr Surg 2008;35:589-96.

5. Tremolet de Villers Y. Nasal fractures. J Trauma 1975;15:
319-27.

6. Fry HJ. Interlocked stresses in human nasal septal cartilage. Br J Plast Surg 1966;19:276-8.

7. Higuera S, Lee EI, Cole P, et al. Nasal trauma and the deviated nose. Plast Reconstr Surg 2007;120:64S-75S.

8. Kim JH, Park SH, Chu HH, et al. The postoperative analysis of closed reducted nasal bone fracture. J Korean Soc Plast Reconstr Surg 1997;24:1343-51. 